\title{
Comparison of Three Methods for Calculating the Standard Deviation of the Wind Direction
}

\author{
D. BRUCE TURNER* \\ Meteorology and Assessment Division, Atmospheric Sciences Research Laboratory, \\ Environmental Protection Agency, Research Triangle Park, NC 27711.
}

(Manuscript received 26 July 1985, in final form 23 November 1985)

\begin{abstract}
Three methods to calculate wind direction standard deviation are evaluated. Although eight hours of wind data show no significant differences between the methods, synthetically generated data having standard deviations near the maximum possible show the Yamartino method to perform well. Also, the wind direction for periods such as an hour is shown to be well represented by the arctangent of the mean sines and cosines.
\end{abstract}

\section{Introduction}

To realistically estimate concentrations from air pollutant sources using air quality simulation models, it is important to represent the atmospheric physics as closely as possible. Therefore, the Meteorology and Assessment Division has advocated the use of fluctuation statistics to estimate atmospheric dispersion (Turner, 1979; Irwin, 1983). Toward this end, it is desirable to calculate $\sigma_{a}$ using a method that accurately gives the root-mean-square deviations of the instantaneous wind direction values used.

Since wind direction is a circular function with a crossover point between $360^{\circ}$ and $0^{\circ}$, standard statistical methods for linear data sets can not be used directly for calculating the standard deviation of wind azimuth $\sigma_{a}$. Statistical methodology for circular data

\footnotetext{
* Permanent affiliation: National Oceanic and Atmospheric Administration, United States Department of Commerce.
}

(Mardia, 1972; Fisher, 1983), relates to the underlying probability density function interpretation of $\sigma_{a}$; this is an interpretation which differs from the meteorologist's definition of $\sigma_{a}$ as derived from wind measuring instrumentation, particularly at large $\sigma_{a}$. In this paper, three published methods are evaluated using two types of data sets.

\section{Available methods}

If all instantaneous values over the time period for which the standard deviation is calculated are retained until the end of the period $p$, a straightforward method (Irwin, 1980) can be used to account for the crossover. The resultant direction is determined and subtracted from $180^{\circ}$. This adjustment is then added to each individual value and the sum and sum of squares using these values determined again. The standard deviation can then be determined directly from this new sum and sum of squares using Eqs. (1) and (2). Of course,

TABLE 1. Data collected and preliminary calculations.

I. Summations of instantaneous values accumulated over the selected time period (for example, 1 hour)

$$
\sum \sin a, \quad \sum \cos a, \quad \sum \sin ^{2} a, \quad \sum \cos ^{2} a, \quad N, \quad \sum \sin a \cos a
$$

II. Calculations made for both Ackermann and Verrall-Williams techniques

$$
\begin{gathered}
\overline{\sin a}=\sum \sin a / N, \quad \overline{\cos a}=\sum \cos a / N, \quad \bar{a}=\arctan (\overline{\sin a}, \overline{\cos a}) \\
\sigma_{s}{ }^{2}=\frac{\sum \sin ^{2} a}{N}-(\overline{\sin a})^{2} \quad \sigma_{c}{ }^{2}=\frac{\sum \cos ^{2} a}{N}-(\overline{\cos a})^{2}
\end{gathered}
$$


TABLE 2. Calculations made for Ackermann method.

$$
\begin{gathered}
\sigma_{s c}=\frac{\sum \sin a \cos a}{N}-\overline{\sin a} \overline{\cos a} \\
\sigma_{a}=\frac{\left[(\overline{\cos a})^{2} \sigma_{s}^{2}+(\overline{\sin a})^{2} \sigma_{c}{ }^{2}-2 \overline{\sin a} \overline{\cos a} \sigma_{s c}\right]^{1 / 2}}{(\overline{\sin a})^{2}+(\overline{\cos a})^{2}}
\end{gathered}
$$

the principal disadvantage of this method is the requirement for intermediate storage of each individual value. A variation of this procedure, but still requiring hundreds of storage locations, has been suggested by Nelson (1984).

Three other methods (Verrall and Williams, 1982; Ackermann, 1983; Yamartino, 1984) overcome these storage requirements provided that the on-line processor can determine the trigonometric functions, sine and cosine, of each instantaneous value. Experimentally, the following five summations are collected over the period $p$ (frequently an hour):

$$
\sum \sin a, \quad \sum \cos a, \quad \sum \sin ^{2} a, \quad \sum \cos ^{2} a, \quad N,
$$

where $a$ is the instantaneous wind direction value and $N$ is the number of instantaneous values used over the time period $p$. All five sums are needed for the VerrallWilliams approach. In addition to these five values, the Ackermann method also requires $\Sigma \sin a \cos a$. The method of Yamartino requires only $\Sigma \sin a, \Sigma \cos a$ and $N$. Note that calculations discussed here use only the wind direction; the wind speed is not used. Using these summations, the standard deviation of wind azimuth, $\sigma_{a}$, is calculated using the equations given in Tables 1-4.

\section{Testing with data from Boulder Atmospheric Ob- servatory tower}

Data from sonic anemometers on the Boulder Atmospheric Observatory tower (Kaimal and Gaynor, 1983) were made available to the author. Instantaneous values of $u, v, w$ at $0.1-\mathrm{sec}$ intervals for one hour were available for eight hours as eight data sets. The wind direction was calculated from each $u$, v pair and corrected for the orientation of the sonic system to yield the wind angle with respect to true north:

$$
a=\arctan (u / v)+\text { correcticin. }
$$

The various trigonometric sums discussed in section 2 were then computed.

The distribution of the $a$ 's, to the rearest degree, were also tabulated and printed at the conclusion of the processing for each data set to determine if crossover between $360^{\circ}$ and $0^{\circ}$ would affect the mean and standard deviation calculated by the standard statistical methods:

$$
\begin{aligned}
a & =\sum a / N, \\
\sigma_{a} & =\left[\left(\sum a^{2} / N\right)-\bar{a}^{2}\right]^{0.5} .
\end{aligned}
$$

If inspection indicated that the stanclard statistical computations would be affected, a second run was made adding an appropriate rotation angle to each observation, making the calculation, and then subtracting the rotation from the calculated mean. Four of the eight data sets required the rotation in order to appropriately apply (1-2). FORTRAN statements used to apply the three methods are shown in the Appendix.

Table 5 compares $\bar{a}$ calculated using the equation given in Table 1 (the resultant of the mean sines and cosines) with $\bar{a}$ calculated using equation (1) for the eight data sets. The values from (1) are those obtained after any required rotation to avoid cross-over and therefore are assumed correct. The values are shown to three decimal digits only to allow comparison of the calculational methods, but are not significant to this degree, since it is rare that a wind system can be oriented to yield direction with greater accuracy than about $5^{\circ}$. No difference between the two methods is

\begin{tabular}{|c|c|c|}
\hline $\begin{array}{l}\text { I. The east and north components of } \\
\text { vector } 1 \text { are }\end{array}$ & $\begin{array}{l}\text { II. The east and north components of } \\
\text { vector } 2 \text { are }\end{array}$ & $\begin{array}{l}\text { III. The magnitude of vectors } \\
1 \text { and } 2 \text { are }\end{array}$ \\
\hline$E_{1}=|\overline{\sin a}|-\sigma_{s}$ & $E_{2}=|\overline{\sin a}|+\sigma_{s}$ & $M_{1}+\left[E_{1}^{2}+N_{1}^{2}\right]^{1 / 2}$ \\
\hline$N_{1}=|\overline{\cos a}|+\sigma_{c}$ & $N_{2}=|\overline{\cos a}|-\sigma_{c}$ & $M_{2}=\left[E_{2}^{2}+N_{2}^{2}\right]^{1 / 2}$ \\
\hline & IV. The dot product of vectors 1 and 2 is & . \\
\hline & $D=E_{1} E_{2}+N_{1} N_{2}$ & \\
\hline & $\sigma_{a}=0.5 \arccos \left[D /\left(M_{1} M_{2}\right)\right]$ & \\
\hline
\end{tabular}
greater than $0.1^{\circ}$.

Table 6 compares $\sigma_{a}$ calculated from (2) with $\sigma_{a}$ 's estimated using the three methods. The maximum error (Verrall-Williams, data set 1 .) is less than $0.5^{\circ}$.

TABLE 3. Calculations made for Verrall-Williams method. 
TABLE 4. Calculations made for the Yamartino method.

\begin{aligned} & \hline$\epsilon=\left\{1 .-\left[(\overline{\sin a})^{2}+(\overline{\cos a})^{2}\right]\right\}^{0.5} \\ & \sigma_{a}=\arcsin (\epsilon)\left[1 .+0.1547 \epsilon^{3}\right] \\ &$\hline\end{aligned}

TABLE 5. Average direction and average direction calculated from mean sines and cosines.

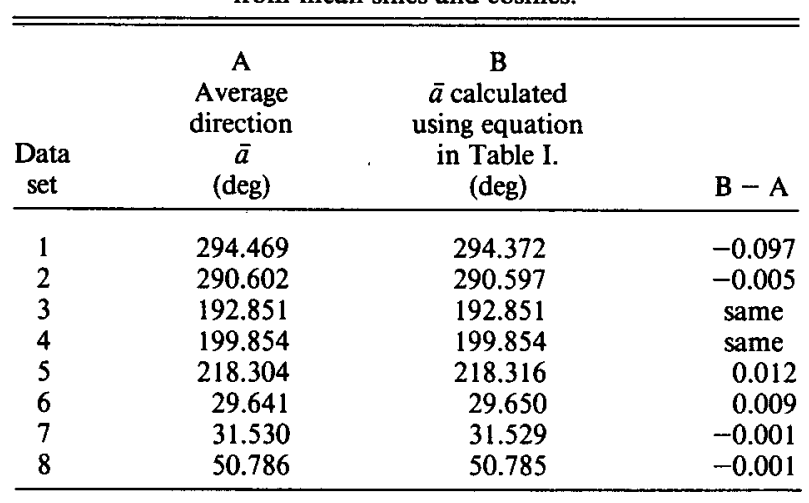

This is less than $2 \%$. From the data available in these eight sets, there is little basis for choosing one of the methods over the other.

\section{Testing using synthesized data}

Yamartino (1984) pointed out that the Ackermann method overestimates $\sigma_{a}$ and the Verrall-Williams method underestimates $\sigma_{a}$ for values greater than about $30^{\circ}$. The maximum standard deviation from the available eight data sets was less than $22^{\circ}$. Low wind speeds and major wind shifts, such as caused by frontal passages and transition from land to sea breeze and vice versa, contribute to large $\sigma_{a}$ values. It has been brought to my attention (Lambeth, personal communication, 1984) that in situations where the surface roughness is large and where there are significant terrain features, mechanical turbulence may cause large directional fluctuations which, in turn, produce large standard deviations. In the extreme, near-uniform distributions around the compass may result. Yamartino also pointed out the occurrence of large $\sigma_{a}$ in some of the data to which he had access. It therefore seemed desirable to examine the performance of the three methods on some data sets generated to test these extremes.

Using routines available from the International Mathematical and Statistical Libraries (1982), a number of wind direction distributions were generated with a wide variety of $\sigma_{a}$ 's. As the distributions were generated, values of wind direction $<0^{\circ}$ or $>360^{\circ}$ were allowed to "wrap around" these crossover points and be included in the distribution. Thus, at the extremea distribution with $\sigma_{a}=101.7^{\circ}-\mathrm{a}$ nearly circular distribution was generated. The same characteristics as noted by Yamartino (overestimation by Ackermann and underestimation by Verrall-Williams) was seen.

Table 7 shows results from a selection of generated distributions. Data for five distributions (each generated with a different "seed") were analyzed for each mean $\sigma_{a}$ displayed in the Table. The maximum error seen here for the Yamartino method is less than $1.2^{\circ}$ (for $\sigma_{\alpha}=76.5$ ). This is a $1.5 \%$ error and verifies Yamartino's statement that his method "is within $\pm 2 \%$ of the correct" $\sigma_{a}$.

Some additional tests of the methods were made by taking generated distributions, rotating them (so that the mean would be at different azimuths), and applying the methods. Both the Yamartino and Ackermann methods gave the same $\sigma_{a}$, regardless of the location of the mean; i.e., the methods are insensitive to rotations. However, The Verrall-Williams method gave some variation with rotation of the distribution but no more than $3.6^{\circ}$ or $5 \%$ of the $\sigma_{a}$.

TABLE 6 . Directly calculated $\sigma_{a}$ compared with $\sigma_{a}$ from three methods.

\begin{tabular}{|c|c|c|c|c|c|c|c|}
\hline $\begin{array}{c}\text { Data } \\
\text { set }\end{array}$ & $\begin{array}{c}\text { A } \\
\text { Calculated } \\
\sigma_{a} \text { Eq. (2) } \\
\text { (deg) }\end{array}$ & $\begin{array}{c}\text { B } \\
\sigma_{a} \text { from } \\
\text { Ackermann } \\
\text { (deg) }\end{array}$ & $\mathbf{B}-\mathbf{A}$ & $\begin{array}{c}\text { C } \\
\sigma_{a} \text { from } \\
\text { Verrall-Williams } \\
\text { (deg) }\end{array}$ & $C-A$ & $\begin{array}{c}\mathrm{D} \\
\sigma_{a} \text { from } \\
\text { Yamartino } \\
\text { (deg) }\end{array}$ & $\mathbf{D}-\mathbf{A}$ \\
\hline 1 & 21.720 & 21.603 & -0.099 & 21.353 & -0.349 & 21.567 & -0.135 \\
\hline 2 & 16.130 & 15.899 & -0.231 & 15.931 & -0.199 & 16.004 & -0.126 \\
\hline 3 & 6.260 & 6.256 & -0.004 & 6.252 & -0.008 & 6.255 & -0.005 \\
\hline 4 & 3.540 & 3.541 & 0.001 & 3.539 & -0.001 & 3.539 & -0.001 \\
\hline 5 & 6.020 & 6.011 & -0.009 & 6.003 & -0.017 & 6.013 & -0.007 \\
\hline 6 & 9.191 & 9.193 & 0.002 & 9.171 & -0.020 & 9.177 & -0.014 \\
\hline 7 & 4.423 & 4.425 & 0.002 & 4.421 & -0.002 & 4.422 & -0.001 \\
\hline 8 & 9.509 & 9.545 & 0.036 & 9.496 & -0.013 & 9.503 & -0.006 \\
\hline \multicolumn{3}{|c|}{ Mean Absolute Error: } & 0.048 & & 0.076 & & 0.037 \\
\hline
\end{tabular}


TABLE 7. Performance of three $\sigma_{a}$ methods using generated distributions.

\begin{tabular}{|c|c|c|c|c|}
\hline \multirow[b]{2}{*}{ Mean $\sigma_{a}$} & \multirow[b]{2}{*}{ Statistic } & \multicolumn{3}{|c|}{$\sigma_{a}$ method } \\
\hline & & Ackermann & Verrall-Williams & Yamartino \\
\hline 9.990 & $\begin{array}{l}\operatorname{maximum} \text { error }\left({ }^{\circ}\right) \\
\text { minimum error }\left(^{\circ}\right) \\
\text { MAE (mean absolute error) }\left({ }^{\circ}\right) \\
\% \text { error (of the MAE) }(\%)\end{array}$ & $\begin{aligned} 0.004 \\
-0.007 \\
0.005 \\
0.05\end{aligned}$ & $\begin{array}{c}-0.092 \\
-0.096 \\
0.094 \\
0.9\end{array}$ & $\begin{array}{l}-0.017 \\
-0.019 \\
0.018 \\
0.2\end{array}$ \\
\hline 19.968 & $\begin{array}{l}\text { maximum error }\left({ }^{\circ}\right) \\
\text { minimum error }\left({ }^{\circ}\right) \\
\text { MAE (mean absolute error) }\left({ }^{\circ}\right) \\
\% \text { error (of the MAE) }(\%)\end{array}$ & $\begin{array}{r}0.053 \\
-0.031 \\
0.024 \\
0.1\end{array}$ & $\begin{array}{c}-0.594 \\
-0.622 \\
0.612 \\
3.1\end{array}$ & $\begin{array}{l}-0.077 \\
-0.097 \\
0.091 \\
0.5\end{array}$ \\
\hline 29.960 & $\begin{array}{l}\operatorname{maximum} \text { error }\left({ }^{\circ}\right) \\
\text { minimum error }\left(^{\circ}\right) \\
\text { MAE (mean absolute error) }\left(^{\circ}\right) \\
\% \text { error (of the MAE) }(\%)\end{array}$ & $\begin{array}{l}0.285 \\
0.010 \\
0.104 \\
0.3\end{array}$ & $\begin{array}{l}-1.446 \\
-1.531 \\
1.500 \\
5.0\end{array}$ & $\begin{array}{l}-0.125 \\
-0.196 \\
0.172 \\
0.6\end{array}$ \\
\hline 49.886 & $\begin{array}{l}\operatorname{maximum} \text { error }\left({ }^{\circ}\right) \\
\text { minimum error }\left(^{\circ}\right) \\
\text { MAE (mean absolute error) }\left(^{\circ}\right) \\
\% \text { error (of the MAE) }(\%)\end{array}$ & $\begin{array}{l}2.911 \\
1.665 \\
2.139 \\
4.3\end{array}$ & $\begin{array}{l}-2.694 \\
-2.993 \\
2.876 \\
5.8\end{array}$ & $\begin{array}{l}-0.145 \\
-.476 \\
0.355 \\
0.7\end{array}$ \\
\hline 76.543 & $\begin{array}{l}\operatorname{maximum} \text { error }\left({ }^{\circ}\right) \\
\text { minimum error }\left(^{\circ}\right) \\
\text { MAE (mean absolute error) }\left(^{\circ}\right) \\
\% \text { error (of the MAE) }(\%)\end{array}$ & $\begin{array}{l}31.759 \\
25.067 \\
27.952 \\
36.5\end{array}$ & $\begin{array}{l}-4.909 \\
-5.703 \\
5.452 \\
7.1\end{array}$ & $\begin{array}{l}-0.480 \\
-1.155 \\
0.819 \\
1.0\end{array}$ \\
\hline 101.685 & $\begin{array}{l}\operatorname{maximum} \text { error }\left(^{\circ}\right) \\
\text { minimum error }\left(^{\circ}\right) \\
\text { MAE (mean absolute error) }\left(^{\circ}\right) \\
\% \text { error (of the MAE) }\left(^{\circ}\right)\end{array}$ & $\begin{array}{r}2617.153 \\
628.747 \\
1516.153 \\
1491\end{array}$ & $\begin{array}{c}-13.004 \\
-14.471 \\
13.741 \\
13.5\end{array}$ & $\begin{array}{c}-0.255 \\
-0.747 \\
0.489 \\
0.5\end{array}$ \\
\hline
\end{tabular}

\section{Conclusion}

The method of estimation of $\sigma_{a}$ by Yamartino (1984) which requires only three accumulators over a given time period and seems readily adaptable to on-line data processing gives excellent results over the entire range of possible standard deviations $0^{\circ}$ to $103.9^{\circ}$. (Here, 102.6 was tested.) The approximation of the mean wind direction by the arctangent of the mean sines and mean cosines also gives excellent results; errors are $<1^{\circ}$ for distributions with $\sigma_{a}<75^{\circ}$. Distributions having $\sigma_{a}$ $>75^{\circ}$ are approaching circular distributions and for these, mean wind direction has little meaning.

Acknowledgments. The author is grateful for the existence of the NOAA Boulder Atmospheric Observatory Tower and the care taken by the individuals maintaining that facility and checking the data from it. The assistance of Jim Wilczak in furnishing wind direction data sets from the NOAA BOA Tower is appreciated. The prompt reply by Messrs. Verrall and Williams to my inquiry, resulting in the addition of the absolute value notations to their equations is also appreciated. The secretarial assistance of Sylvia Coltrane is gratefully acknowledged.

\section{APPENDIX}

FORTRAN Subroutines for Three Sigma Methods

In the main program accumulators are initialized:

$$
\begin{aligned}
& \mathrm{AN}=0 \text {. } \\
& \mathrm{SS}=0 \text {. } \\
& \mathrm{SC}=0 \text {. } \\
& \mathrm{SSS}=0 \text {. } \\
& \mathrm{SCC}=0 \text {. } \\
& \mathrm{SSC}=0 \text {. } \\
& \text { C AN IS THE NUMBER OF VALUES } \\
& \text { C SS IS THE SUM OF SINES } \\
& \text { C SC IS THE SUM OF COSINES } \\
& \text { C SSS IS THE SUM OF SINES SQUARED } \\
& \text { C SCC IS THE SUM OF COSINES SQUARED } \\
& \text { C SSC IS THE SUM OF SINE TIMES COSINE }
\end{aligned}
$$

As each instantaneous value of wind direction $A$ is obtained, the following is accomplished:

$$
\begin{array}{ll}
\mathrm{AN}=\mathrm{AN}+1 \\
\text { CONVERT ANGLE TO RADIANS } \\
\mathrm{A}=\mathrm{A} / 57.29578 \\
\mathrm{~S}=\mathrm{SIN}(\mathrm{A}) \\
\mathrm{C}=\mathrm{COS}(\mathrm{A}) \\
\mathrm{SS}=\mathrm{SS}+\mathrm{S}
\end{array}
$$




$$
\begin{aligned}
& \mathrm{SC}=\mathrm{SC}+\mathrm{C} \\
& \mathrm{SSS}=\mathrm{SSS}+\mathrm{S} * \mathrm{~S} \\
& \mathrm{SCC}=\mathrm{SCC}+\mathrm{C} * \mathrm{C} \\
& \mathrm{SSC}=\mathrm{SSC}+\mathrm{S} * \mathrm{C}
\end{aligned}
$$

At the conclusion of obtaining all instantaneous values over the time interval, $p$, the mean wind direction is approximated using the function:

$$
\text { ABAR }=\text { ARES(SS,SC) }
$$

and the following calls are made to the methods:

CALL ACKER(AN,SS,SC,SSS,SCC,SSC, SIGD)

CALL VERWIL(AN,SS,SC,SSS,SCC, SIGA)

CALL YATINO(AN,SS,SC,SIGTH)

The $\sigma_{a}$ is SIGD, SIGA, and SIGTH from the Ackermann, Verrall-Williams, and Yamartino methods respectively.

The function for determining mean direction is

FUNCTION ARES(SS,SC)
ARES $=$ ATAN2(SS,SC)
ARES $=57.29578 *$ ARES
IF(ARES.LT.O.) ARES = ARES + 360.
RETURN
END

The subroutines are

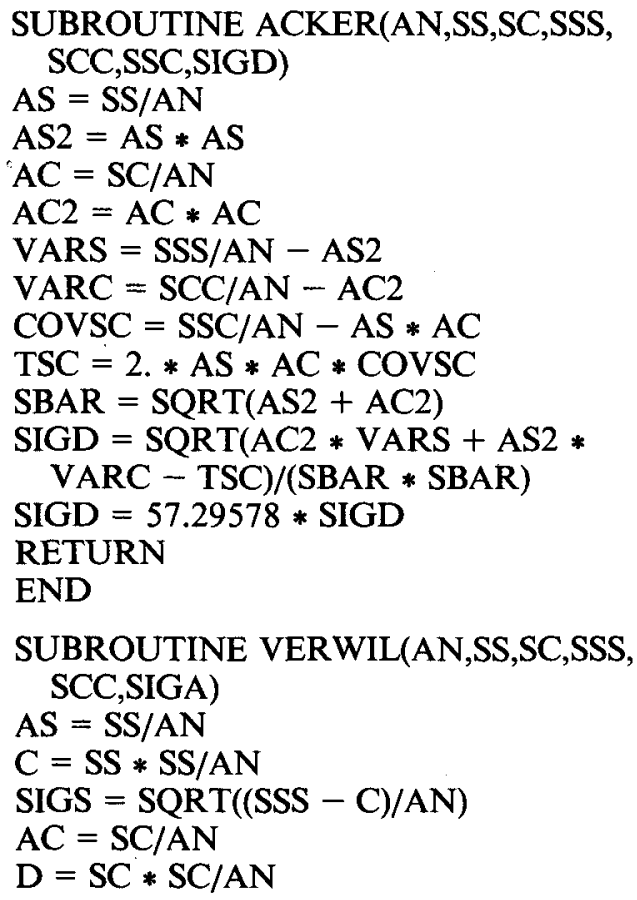

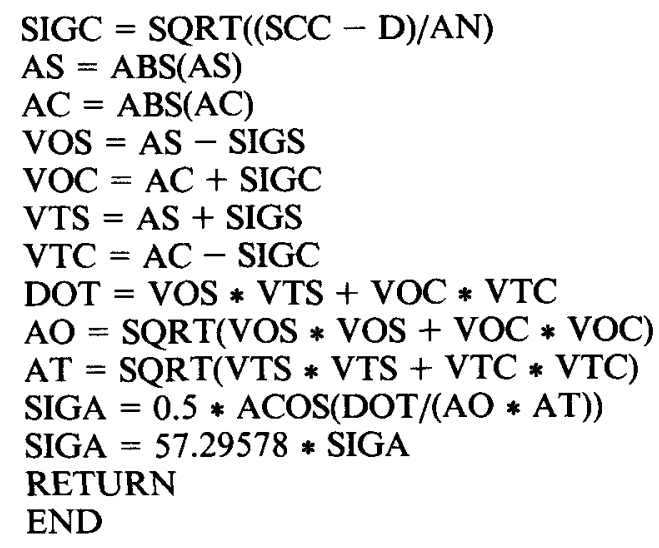

SUBROUTINE YATINO(AN,SS,SC, SIGTH)

$\mathrm{AS}=\mathrm{SS} / \mathrm{AN}$

$\mathrm{AC}=\mathrm{SC} / \mathrm{AN}$

$\mathrm{E}=\mathrm{SQRT}(1 .-(\mathrm{AS} * \mathrm{AS}+\mathrm{AC} * \mathrm{AC}))$

$\mathrm{SIGTH}=\operatorname{ASIN}(\mathrm{E}) *(1 .+0.1547 * \mathrm{E} * * 3$.

SIGTH $=57.29578 *$ SIGTH

RETURN

END 